\title{
DESEMPENHO DAS EXPORTAÇÕES: INFLUÊNCIA DOS RECURSOS INTANGÍVEIS, CAPACIDADES DINÂMICAS E ORIENTAÇÃO EMPREENDEDORA
}

EXPORT PERFORMANCE: INFLUENCE OF INTANGIBLE RESOURCES, DYNAMIC CAPABILITIES AND ENTREPRENEURIAL

\section{RESULTADOS DE EXPORTACIÓN: INFLUENCIA DE PATRIMONIO RECURSOS, CAPACIDADES DINÁMICAS Y ORIENTACIÓN EMPRESARIAL}

\author{
Albertina Paula Monteiro \\ Doutora em Marketing e Estratégia pela Universidade do Minho, Portugal \\ Professora de Contabilidade e Gestão no Instituto Politécnico do Porto (ISCAP) e no Instituto \\ Superior de Administração e Gestão (ISAG), Portugal \\ E-mail: amonteiro@iscap.ipp.pt (Portugal)

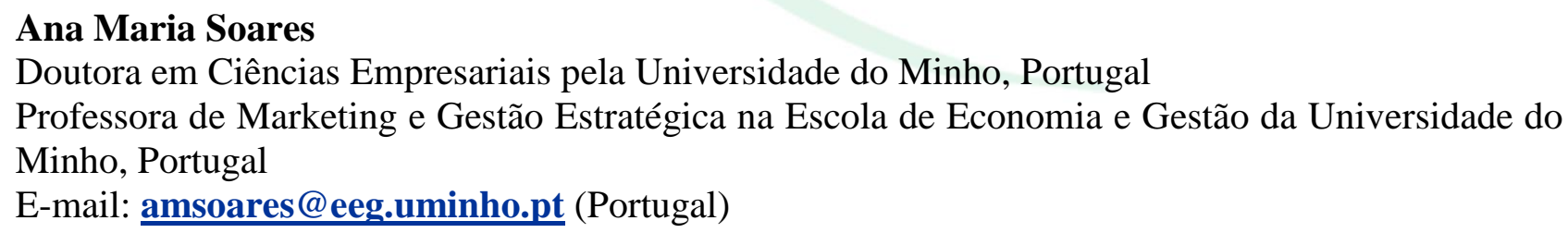




\title{
DESEMPENHO DAS EXPORTAÇÕES: INFLUÊNCIA DOS RECURSOS INTANGÍVEIS, CAPACIDADES DINÂMICAS E ORIENTAÇÃO EMPREENDEDORA
}

\section{RESUMO}

Compreender de forma substancial os determinantes que influenciam o desempenho internacional, em particular as exportações, é vital para a sobrevivência e expansão das empresas e para o consequente crescimento económico de países exportadores que estão fortemente dependentes dos mercados internacionais. Porquanto, este estudo centra-se sobretudo na forma como os recursos e capacidades permitem às empresas alcançarem vantagem competitiva e desempenho superior em mercados externos. Esta investigação visa comprovar a premissa base da Resource-Based View (RBV) e da Dynamic Capabilities View (DCV), segundo as quais os recursos intangíveis de uma empresa influenciam o desenvolvimento de capacidades importantes para o desempenho das exportações. Assim, desenvolvemos um modelo que avalia o impacto dos relacionamentos empresariais e do conhecimento na orientação empreendedora e nas capacidades dinâmicas, bem como no desempenho das exportações. Os resultados de um questionário a uma amostra de 265 empresas exportadoras do norte de Portugal permitem confirmar seis das dez hipóteses propostas, permitindo concluir que o desempenho das exportações é influenciado de forma direta pelas capacidades dinâmicas e pela orientação empreendedora e de forma indireta pelo conhecimento e relacionamentos empresariais. Contrariamente às hipóteses formuladas, a orientação empreendedora não é influenciada diretamente pelos relacionamentos nem pelos conhecimentos das empresas mas indiretamente através das capacidades dinâmicas.

Palavras-chave: Desempenho das Exportações; Recursos e Capacidades; Capacidades Dinâmicas; Orientação Empreendedora.

\section{EXPORT PERFORMANCE: INFLUENCE OF INTANGIBLE RESOURCES, DYNAMIC CAPABILITIES AND ENTREPRENEURIAL}

\begin{abstract}
Understanding the determinants that influence international performance, in particular exports, is vital to the survival and expansion of enterprises and the consequent economic growth of exporting countries that are heavily dependent of international markets. Thus this study focuses mainly on how resources and capabilities enable companies to achieve competitive advantage and superior performance in foreign markets. This research builds upon the Resource-Based View (RBV) and Dynamic Capabilities View (DCV) premise that companies' intangible resources influence the development of important capabilities to export performance. Thus, we developed and tested a model entailing knowledge and business relationships positive impact on entrepreneurial orientation and dynamic capabilities, which translates positively in exports' performance. Results of a survey of a sample of 265 exporting companies in the north of Portugal confirm six out of the ten proposed hypothesis and confirm that export performance is directly impacted by dynamic capabilities and entrepreneurial orientation and indirectly by knowledge and business relationships. Contrary to hypothesized, entrepreneurial orientation is influenced directly only by dynamic capabilities, as business relationships and knowledge do not have a direct significant impact but indirectly through dynamic capabilities.
\end{abstract}

Keywords: Export Performance; RBV; Dynamic Capabilities; Entrepreneurial Orientation; Internationalization. 
Albertina Paula Monteiro, Ana Maria Soares \& Orlando Lima Rua

\section{RESULTADOS DE EXPORTACIÓN: INFLUENCIA DE PATRIMONIO RECURSOS, CAPACIDADES DINÁMICAS Y ORIENTACIÓN EMPRESARIAL}

\section{RESUMEN}

Entender sustancialmente los determinantes que influyen en el rendimiento internacional, en particular las exportaciones, es vital para la supervivencia y la expansión de las empresas y el consiguiente crecimiento económico de los países exportadores que dependen en gran medida de los mercados internacionales. Debido a que este estudio se centra principalmente en cómo los recursos y capacidades permiten a las empresas para lograr una ventaja competitiva y un rendimiento superior en los mercados extranjeros. Esta investigación tiene como objetivo demostrar la premisa básica de la vista basada en recursos ( RBV) y las capacidades dinámicas view (dcv), según el cual los activos intangibles de una influencia organización el desarrollo de una capacidad considerable para los resultados de exportación. Por lo tanto, hemos desarrollado un modelo que evalúa el impacto de las relaciones comerciales y el conocimiento en la orientación emprendedora y las capacidades dinámicas y resultados de exportación. Los resultados de un cuestionario a una muestra de 265 empresas exportadoras en el norte de portugal nos permiten confirmar seis de las diez hipótesis planteadas, lo que permite concluir que los resultados de exportación es influenciado directamente por las capacidades dinámicas y la orientación emprendedora e indirectamente a través de los conocimientos y relaciones de negocios. Contrariamente a las hipótesis, la orientación emprendedora no está directamente influenciada por las relaciones o conocimientos de las empresas, sino indirectamente a través de las capacidades dinámicas.

Palabras-clave: Comportamiento de las Exportaciones; Los Recursos y Capacidades; Las Capacidades Dinámicas; Orientación Emprendedora. 
Desempenho das Exportações: Influência dos Recursos Intangíveis, Capacidades Dinâmicas e

Orientação Empreendedora

\section{INTRODUÇÃO}

A prosperidade social e económica de pequenas economias depende fortemente do comércio internacional. Assim, para o desenvolvimento da economia e sobrevivência e/ou expansão das empresas em mercados cada vez mais competitivos, torna-se fundamental a dinamização da internacionalização. Gestores de empresas com atividades internacionais têm que competir com a sua melhor capacidade, atendendo aos recursos disponíveis, nos mercados externos (Morgan, Vorhies e Schlegelmilch, 2006). Segundo a teoria Resource-Based View (RBV), a posse de recursos estratégicos permite às empresas a obtenção de vantagens competitivas. No entanto, pesquisas recentes têm-se focalizado nos recursos intangíveis por serem mais importantes sob o ponto de vista estratégico e mais relevantes para o desempenho empresarial (Morgan et al., 2006; Bakar e Ahma, 2010). Por outro lado, a investigação tem-se centrado nas capacidades dinâmicas como fonte de vantagem competitiva sustentável (Teece, 2007; Wu, 2010; López, 2005; Teece, Pisano e Amy, 1997), conferindo à teoria dos recursos e capacidades uma abordagem mais dinâmica.

Tendo como ponto de partida a RBV e a Dynamic Capabilities View (DCV), este estudo pretende avaliar a interação entre recursos e capacidades (disponíveis para o mercado internacional) e o desempenho das exportações, através da análise do efeito mediador das capacidades dinâmicas e da orientação empreendedora.

A opção por esta temática deve-se sobretudo à necessidade de as empresas compreenderem o processo que permite alcançar desempenho superior a nível das exportações utilizando e/ou reforçando estratégias empreendedoras, assim como desenvolvendo outras capacidades, em particular capacidades dinâmicas. A melhoria do desempenho das exportações é crucial dado que estimula o crescimento empresarial e garante a sobrevivência das empresas a longo prazo, contribuindo, desta forma, para o crescimento económico (Sousa, Martínez-López e Coelho, 2008). A cabal compreensão dos determinantes do desempenho das exportações é da maior relevância para a definição de políticas públicas, para gestores e para os investigadores nesta área. 


\section{FUNDAMENTAÇÃO TEÓRICA}

\subsection{RECURSOS E CAPACIDADES EMPRESARIAIS}

A RBV, uma das teorias mais conceituadas em gestão estratégica, desenvolve-se a partir da premissa segundo a qual os recursos e capacidades estratégicos permitem às empresas obter vantagens competitivas (Wernerfelt, 1984; Barney, 1991). Barney (1991, p. 102) define vantagem competitiva como a "implementação estratégica que permita a criação de valor e não seja, simultaneamente, implementada por todos os concorrentes atuais ou potenciais", assinalando que vantagem competitiva não pode existir para empresas idênticas. Este autor sublinha que a teoria RBV se baseia na suposição de que os recursos/capacidades desenvolvidos e controlados pela empresa devem ser raros, valiosos, insubstituíveis e de difícil imitação (Barney, 1991). No entanto, a heterogeneidade e imobilidade são condições básicas mas não suficientes para obter vantagem competitiva sustentável (Barney, Wright e Ketchen, 2001).

Os recursos são entendidos como ativos tangíveis ou intangíveis que uma empresa possui, controla ou tem acesso de forma semi-permanente (Helfat e Peteraf, 2003). Existem diferentes tipologias de recursos, tais como tecnológicos, financeiros, humanos, físicos e organizacionais (Loane e Bell, 2006; Bakar e Ahmad, 2010; Morgan et al. (2006). Para Wernerfelt (1984), o conceito de recurso é limitado aos atributos que aumentam a eficiência e a eficácia da empresa. Por outro lado, os recursos devem ter alguma capacidade de gerar lucros ou evitar prejuízos (Miller e Shamsie, 1996). Neste sentido, os recursos não abrangem apenas os bens de uma organização mas também as suas capacidades (Henderson e Cockburn, 1994).

A capacidade refere-se à aptidão de uma empresa realizar um conjunto coordenado de tarefas, utilizando os recursos organizacionais, a fim de alcançar um resultado específico (Helfat e Peteraf, 2003). Segundo Amit e Schoemaker (1993), capacidade designa a aptidão da empresa para mobilizar recursos, geralmente em combinação, usando os processos organizacionais, para um efeito final desejado. Segundo estes autores, as capacidades são desenvolvidas nas áreas funcionais ou pela combinação de recursos físicos, humanos e/ou tecnológicos controlados pela empresa.

Na literatura, verifica-se que tem vindo a aumentar a importância atribuída aos recursos intangíveis. Morgan et al. (2006), enfatizam a importância de seis tipos de recursos intangíveis para o desempenho das exportações, nomeadamente, reputação, financeiros, informação/conhecimento, cultura, humanos e relacionamentos. Aliás, Madsen, Alsos, Borch, Ljunggren e Brastad (2007), centram-se especificamente no papel dos recursos intangíveis (relacionamentos e conhecimento) e capacidades dinâmicas no desenvolvimento da orientação empreendedora. 
Desempenho das Exportações: Influência dos Recursos Intangíveis, Capacidades Dinâmicas e

Orientação Empreendedora

\subsection{RELACIONAMENTOS EMPRESARIAIS}

Os relacionamentos empresariais consistem em relações entre a empresa e entidades externas, como, clientes, fornecedores, concorrentes e instituições governamentais (Davis e Mentzer, 2008). Os recursos baseados em relacionamentos são promissores de vantagem competitiva sustentável, na medida em que são recursos distribuídos de forma assimétrica, imperfeitamente móveis, difíceis de imitar e não têm substitutos disponíveis de acordo com Barney (1991). A luta pela vantagem competitiva numa economia globalizada gira, cada vez mais, em torno do valor dos relacionamentos (Davis e Mentzer, 2008). Assim, relacionamentos comprometidos são fundamentais para um desempenho superior (Arndt, 1979; Morgan et al. (2006). Apesar dos relacionamentos comprometidos terem impacto positivo no desempenho, uma empresa deve estabelecer relações não só em função do desempenho esperado, mas também de forma a permitir o aperfeiçoamento de capacidades que permitem desenvolver outros recursos (Arndt, 1979).

\subsection{CONHECIMENTO}

O conhecimento refere-se a qualquer informação, crença ou a aptidão que a empresa pode incorporar nas suas atividades (Anand, Glick e Manz, 2002). A aquisição e exploração do conhecimento foram qualificados como um recurso fundamental para a criação de vantagens competitivas sustentáveis (Grant, 1996; Nahapiet e Ghoshal 1998; Tsai e Ghoshal, 1998). O uso efetivo de informações relevantes, precisas e oportunas é um importante meio para responder a muitos dos problemas das empresas nos mercados externos (Katsikeas e Morgan, 1994). Aliás, a principal barreira para a internacionalização das pequenas empresas consiste na falta de conhecimento (Loane e Bell, 2006).

\subsection{CAPACIDADES DINÂMICAS}

A literatura sugere que a teoria dos recursos e capacidades não explica adequadamente a forma como as empresas alcançam vantagem competitiva em ambientes de mudança rápida e inesperada (Eisenhardt e Martin, 2000; Teece et al., 1997) em que a mudança tecnológica é rápida, a natureza da concorrência e mercados é difícil de determinar e o tempo de colocação do produto no mercado é crítico (Teece et al., 1997). Em mercados versáteis, a empresa deve ter a capacidade de renovar competências de modo a assegurar continuamente a coerência entre o ambiente de negócios 
Albertina Paula Monteiro, Ana Maria Soares \& Orlando Lima Rua

e a estratégia de atuação. Assim, a atenção dos investigadores tem vindo a desviar-se para as capacidades dinâmicas como fonte de vantagem competitiva sustentável (Teece, 2007; Eisenhardt e Martin, 2000; Teece et al., 1997). Teece et al. (1997) definem capacidades dinâmicas como a capacidade da empresa para integrar, construir e reconfigurar competências internas e externas a fim de responder rapidamente às mudanças do atual ambiente de negócios. Para Hung-Hsin e Lee (2009), as capacidades dinâmicas organizacionais são um processo intrínseco evolutivo que pode facilitar a resolução de problemas, melhorar a tomada de decisões, estimular os ideais de criação e ajudar os membros a implementar efetivamente os objetivos organizacionais.

\subsection{ORIENTAÇÃO EMPREENDEDORA}

A orientação empreendedora constituí um dos principais temas de investigação em negócios internacionais, enriquecendo tanto o estudo sobre o empreendedorismo como sobre a internacionalização de empresas (McDouhall e Oviatt, 2000). Para Miller (1983), uma organização com orientação empreendedora aposta em inovação em produtos e/ou mercados, atua com algum risco e de forma proativa perante sua concorrência. De acordo com Avlonitis e Salavou (2007), orientação empreendedora constitui um fenómeno que reflete a capacidade de gestão, através da qual as empresas se envolvem em iniciativas proativas e agressivas para alterar o cenário competitivo no sentido de alcançar vantagem. Pearce, Fritz e Davis (2010) orientação empreendedora como um conjunto de comportamentos distintos relacionados com características alicerçadas na inovação, proatividade, competitividade, capacidade de assunção de riscos e autonomia.

As dimensões que caracterizam a orientação empreendedora têm sido objeto de diversos estudos. Miller (1983) sugere que a orientação empreendedora é formada por três dimensões fundamentais: inovação, assunção do risco e proatividade. Lumpkin e Dess (1996) propõem duas dimensões adicionais, designadamente, agressividade competitiva e autonomia. Assim, segundo estes autores, a orientação empreendedora inclui a tendência para agir de forma autónoma, a vontade de inovar e de assumir riscos e a tendência para ser agressivo para com os concorrentes e proativo relativamente às oportunidades. A literatura indica que as dimensões mais comummente utilizadas em pesquisa empresarial são: inovação, proatividade e assunção de riscos (Kropp, Lindsay e Shoham, 2008). Segundo Miller (1983), só as empresas que possuem elevado nível nas três dimensões de orientação empreendedora (inovação, assunção do risco e proatividade) seriam consideradas como potencialmente empreendedoras. 
Desempenho das Exportações: Influência dos Recursos Intangíveis, Capacidades Dinâmicas e

Orientação Empreendedora

A inovação, definida como o desenvolvimento de soluções criativas e originais para enfrentar ameaças com que se defronta a empresa (Knight, 1997), tornou-se um fator importante para caracterizar empreendedorismo, refletindo uma tendência para a empresa se envolver em novas ideias, novidades, experiência e em processos criativos que podem resultar em novos produtos, serviços ou processos tecnológicos (Lumpkin e Dess, 1996). Em termos de estratégia de diferenciação, as empresas começam a pensar em inovação porque querem oferecer soluções diferentes em diferentes mercados (Vila e Kuster, 2007), trazendo novidade para a empresa e para o mercado. A inovação constitui uma importante dimensão da orientação empreendedora (Lumpkin e Dess, 1996), apesar de envolver maior risco, uma vez que o investimento em inovação pode não ter o retorno esperado. No âmbito da internacionalização, a investigação sugere que a inovação pode ser importante para ganhar vantagem competitiva a nível internacional, dado que muitas empresas internacionais têm maiores oportunidades de aprender, podendo conduzir à inovação (Hitt, Hoskisson e Kim, 1997).

A proatividade, outra dimensão que caracteriza uma orientação empreendedora, refere-se à antecipação de futuros problemas, necessidades ou mudanças (Lumpkin e Dess, 1996). Frank, Kessler e Fink (2010) consideram proatividade a disposição e capacidade da empresa se antecipar às novidades, tão cedo quanto possível, e agir como uma empresa precursora face aos seus concorrentes, ao invés de esperar por novos desenvolvimentos e tendências. Dado o crescente ambiente competitivo global, uma atitude proativa é vista como importante para a sobrevivência e sucesso das empresas (Knight, 1997).

Estratégias inovadoras e proativas geralmente estão associadas à assunção de riscos (Naldi, Nordqvist, Sjöberg e Wiklund, 2007). O conceito de risco pode ter diferentes significados, dependendo do contexto e do nível de análise (Lumpkin e Dess, 1996). Geralmente, o risco está relacionado com a experiência face a incertezas (Vila e Kuster, 2007). Para Zahra (2008), a assunção de risco significa forte disposição para apoiar projetos inovadores e experimentais cujo retorno é incerto. Quando os empresários percecionam menos risco em atividades internacionais, a empresa irá comprometer-se mais com estas operações e avançar no processo de internacionalização (Acedo e Florin, 2006).

\subsection{DESEMPENHO DAS EXPORTAÇÕES}

Com o constante aumento dos negócios e da concorrência a nível internacional, a compreensão dos determinantes do desempenho internacional, essencialmente das exportações, 
tornou-se particularmente importante, contribuindo para o desenvolvimento de várias pesquisas neste âmbito (Sousa et al., 2008). No entanto, a falta de uma base teórica abrangente torna difícil integrar os resultados de diferentes estudos (Morgan et al., 2004; Sousa et al., 2008).

Em particular, o construto de desempenho das exportações tem sido objeto de uma diversidade de abordagens. Na literatura consideram-se dois tipos de indicadores do desempenho das exportações, objetivos e subjetivos (ver Sousa et al., 2008). Os indicadores que se baseiam principalmente em valores absolutos como a intensidade de exportação, o volume de vendas, quota de mercado, entre outros, são denominadas de medidas objetivas, enquanto os indicadores que medem o desempenho percetual ou atitudinal, como o sucesso percebido e a satisfação com as vendas são considerados medidas subjetivas (Sousa et al., 2008).

Sousa et al. (2008) salientam que, dada a natureza multifacetada do conceito de desempenho das exportações, o uso de vários indicadores se torna necessário para uma avaliação fiável. Estes autores assinalam que apesar da utilização de um grande número de diferentes medidas (cerca de 50), apenas algumas foram frequentemente utilizadas, nomeadamente: intensidade de exportação (peso das exportações no total das vendas); crescimento das vendas; lucro; quota de mercado; satisfação com desempenho das exportações em geral e sucesso percebido. Por outro lado, as medidas menos utilizadas são: retorno sobre o investimento; qualidade do relacionamento com o distribuidor; satisfação do cliente e satisfação com a qualidade produto/serviço em relação aos concorrentes.

Outro aspeto importante no estudo no desempenho das exportações é a unidade de análise. Katsikeas, Leonidou e Morgan (2000) e Sousa et al. (2008) verificam que a maioria dos estudos analisados utiliza a empresa como unidade de análise (desempenho das exportações avaliado no contexto das atividades gerais da empresa nos mercados externos), ao passo que para Cavusgil e Zou (1994) a unidade de análise na pesquisa em desempenho das exportações deve ser a export venture, combinação de um único produto ou linha de produtos exportados para o principal mercado, pois as empresas podem ter mais que um produto ou linha de produto e cada um deles pode ter um impacto diferente no desempenho das exportações (Sousa et al., 2008). Para Piercy, Kaleka e Katsikeas (1998), o foco na export venture contribui para uma avaliação mais precisa dos fatores associados com desempenho superior a nível das exportações pois em muitas empresas os gestores desenvolvem uma estratégia de marketing apenas para o principal mercado, ficando os mercados secundários sem estratégia definida ou sujeitos à estratégia definida para o principal mercado (Lages e Montgomery, 2004). 
Desempenho das Exportações: Influência dos Recursos Intangíveis, Capacidades Dinâmicas e Orientação Empreendedora

O estudo desenvolvido por Morgan et al. (2004) revela que o desempenho das exportações está fortemente relacionado com a vantagem posicional da empresa no mercado internacional e que esta está diretamente relacionada com a disponibilidade de recursos e capacidades para os mercados externos. Por seu turno, Dhanaraj e Beamish (2003) concluem que os recursos são bons preditores de estratégia de exportação (operacionalizada em termos de grau de envolvimento em mercados externos) e que esta influencia positivamente o desempenho das exportações.

\section{MODELO E HIPÓTESES DE INVESTIGAÇÃO}

Este estudo pretende analisar a relação entre os diferentes recursos intangíveis e capacidades disponíveis para o principal mercado internacional no desempenho das exportações. Especificamente, centramo-nos no papel dos recursos intangíveis (relacionamentos empresariais e conhecimento) e capacidades dinâmicas no desenvolvimento de orientação empreendedora. Este estudo objetiva ainda avaliar o impacto destes recursos e capacidades no desempenho das exportações. Adicionalmente, visa avaliar o efeito mediador das capacidades dinâmicas e orientação empreendedora entre recursos intangíveis e desempenho das exportações. A figura 1 presenta o modelo de pesquisa.

Figura 1- Modelo de pesquisa

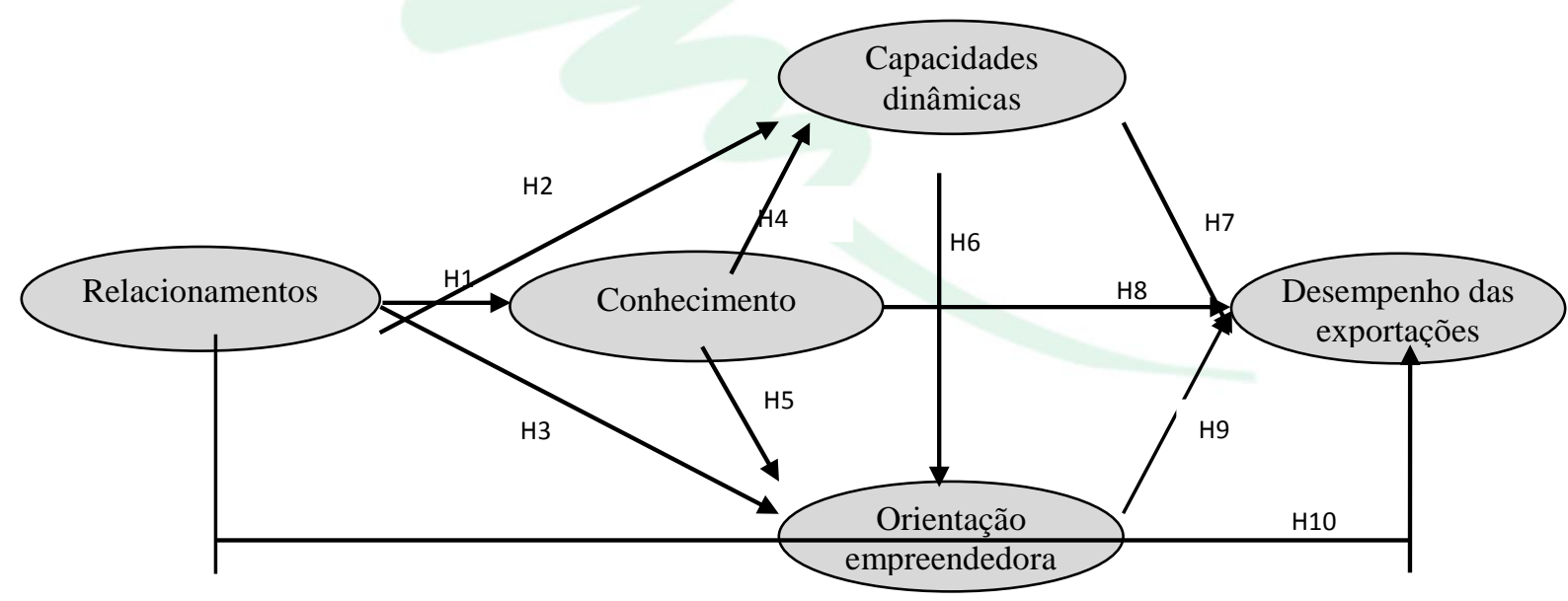


Albertina Paula Monteiro, Ana Maria Soares \& Orlando Lima Rua

\subsection{RELAÇÃO ENTRE OS DIFERENTES RECURSOS INTANGÍVEIS E CAPACIDADES}

A literatura sugere que os recursos e capacidades estão relacionados entre si (Dhanaraj e Beamish, 2003; Morgan et al., 2004). Gulati, Nohria e Zaheer (2000) sustentam que os relacionamentos permitem às empresas o acesso a informações e a outros recursos. Em contextos internacionais, a informação é um recurso que está relacionado com conhecimento do mercado externo, clientes, concorrentes e distribuidores (Morgan et al., 2006). Assim, propõe-se a seguinte hipótese de trabalho:

H1: Os relacionamentos empresariais têm um efeito positivo no nível de conhecimento.

Para Zahra, Sapienza e Davidsson (2006), as capacidades dinâmicas entendem-se como a aptidão de reconfigurar os recursos e rotinas de uma empresa de forma a serem uteis à tomada de decisão. Assim, o desenvolvimento de capacidades dinâmicas depende dos recursos disponíveis nas empresas (Madsen et al., 2007). De facto, Wu e Wang (2007) sublinham que a integração, reconfiguração e aprendizagem de recursos só se tornam significativos quando os próprios recursos são abundantes. Adicionalmente a literatura sugere que a orientação empreendedora depende dos recursos internos da empresa (Wiklund e Shepherd, 2005; Madsen et al., 2007).

Neste contexto, pretende-se analisar o modo como as capacidades dinâmicas organizacionais e orientação empreendedora são aperfeiçoadas pela presença de recursos intangíveis (relacionamentos empresariais e conhecimento). Ou seja:

H2: Os relacionamentos empresariais têm um efeito positivo no desenvolvimento de capacidades dinâmicas.

H3: Os relacionamentos empresariais têm um efeito positivo na orientação empreendedora.

H4:O nível de conhecimento tem um efeito positivo no desenvolvimento de capacidades dinâmicas.

H5: O nível de conhecimento tem um efeito positivo na orientação empreendedora.

Madsen et al. (2007) avaliaram o impacto das capacidades dinâmicas na orientação empreendedora e verificaram uma relação positiva e significativa entre estas duas variáveis. Assim, este estudo pretende também testar a hipótese que se segue.

H6: As capacidades dinâmicas têm um efeito positivo na orientação empreendedora. 
Desempenho das Exportações: Influência dos Recursos Intangíveis, Capacidades Dinâmicas e Orientação Empreendedora

\subsection{RELAÇÃO ENTRE RECURSOS E CAPACIDADES E DESEMPENHO DAS EXPORTAÇÕES}

Teece (2007) argumenta que as capacidades dinâmicas permitem às empresas alcançar desempenho superior a longo prazo. Wu (2006), no âmbito das empresas de base tecnológica e no mercado nacional, encontra uma relação positiva entre capacidades dinâmicas e desempenho. No contexto dos negócios internacionais, pretendemos testar a seguinte hipótese:

H7: As capacidades dinâmicas têm um efeito positivo no desempenho das exportações.

Vários estudos confirmam o papel do conhecimento na obtenção de desempenho superior ao nível das atividades de exportação (Morgan et al., 2006; Tooksoon e Mohamad, 2010). Assim:

H8: O conhecimento têm um efeito positivo no desempenho das exportações.

Uma forte orientação empreendedora pode ser a chave para melhorar o desempenho (Lumpkin e Dess, 1996; Covin e Slevin, 1989; Miller, 1983), sobretudo em ambientes empresariais cada vez mais competitivos (Naldi et al. 2007). Assim, propõe-se que:

H9: A orientação empreendedora tem um efeito positivo no desempenho das exportações.

$\mathrm{Na}$ economia global de hoje, a luta pela vantagem competitiva, cada vez mais, gira em torno do valor dos relacionamentos de uma empresa (Davis e Mentzer, 2008). Por seu turno, a literatura sugere que relacionamentos comprometidos são fundamentais para o desempenho superior (Arndt, 1979; Morgan et al., 2006). Neste sentido, pretende-se testar a última hipótese desta investigação:

H10: Os relacionamentos têm um efeito positivo no desempenho das exportações. 


\section{METODOLOGIA}

\subsection{DESENVOLVIMENTO DO INSTRUMENTO DE MEDIDA}

Este estudo utiliza o inquérito por questionário, à semelhança de muitos dos estudos empíricos no campo do desempenho das exportações (Sousa et al., 2008). O questionário inclui instrumentos validados em estudos anteriores que foram traduzidos para português. A utilização de escalas pré-existentes foi privilegiada de forma a permitir a comparabilidade com estudos anteriores. O questionário foi pré-testado no meio académico e em duas empresas exportadoras. Os participantes foram convidados a destacar dificuldades com o instrumento de análise e fornecer sugestões para a melhoria. O questionário final é composto por três partes. A primeira parte contém informações sobre a empresa e atividade de exportação. A segunda parte inclui questões relacionadas com os recursos intangíveis (relacionamentos e conhecimento), capacidades dinâmicas e orientação empreendedora. Estas variáveis foram avaliadas utilizando, respetivamente, as escalas de Morgan et al. (2006), Wu e Wang (2007) e Covin e Slevin (1989) numa escala de likert de 7 pontos. A terceira e última parte incide sobre o desempenho das exportações. Este construto foi também avaliado numa escala de likert de 7 pontos, recorrendo à escala de Okpara (2009). De referir que a unidade de análise utilizada nesta investigação foi a export venture.

\subsection{CONTEXTO DE PESQUISA, PROCESSO DE RECOLHA DE DADOS E AMOSTRA}

Para testar as hipóteses propostas utilizamos uma amostra de empresas exportadoras portuguesas. A escolha de um único país é consistente com a literatura (Sousa et al., 2008). Adicionalmente a opção pelas empresas portuguesas revela-se interessante face à sua situação económica e à sua forte dependência das exportações (Sousa e Bradley, 2006).

Dado o elevado número de empresas exportadoras registadas no Instituto Nacional de Estatística (17.330) centramo-nos nas empresas da região norte (6.653 registos). Este procedimento é também consistente com a literatura na medida em que diversos estudos restringem a análise a determinadas regiões de um país (Sousa et al., 2008). O questionário foi aplicado online às 1.780 empresas que apresentavam o endereço eletrónico.

O email, com o link de acesso ao questionário, foi endereçado aos responsáveis pelas exportações. Miesenböck (1988) considera que estas pessoas são as mais indicadas para responder a um questionário deste tipo, dado o seu envolvimento e responsabilidade direta nas decisões de 
Desempenho das Exportações: Influência dos Recursos Intangíveis, Capacidades Dinâmicas e Orientação Empreendedora

exportação, e tem sido igualmente a prática da maioria dos estudos realizados neste âmbito (Sousa et al., 2008).

A aplicação dos questionários teve início no final do mês de novembro do ano de 2011, com o envio às 1.780 empresas, tendo sido feito um reenvio posteriormente. Depois, para aumentar a taxa de resposta contatamos por telefone algumas empresas selecionadas aleatoriamente a solicitar a resposta.

Nos contatos realizados, quer na fase de identificação de novos emails (email devolvidos), quer nesta última (contato telefónico a solicitar resposta), foram identificadas 270 empresas que não exportavam (nunca exportaram e, se venderam para o mercado externo foi esporadicamente), prestavam serviços (não incluídas neste estudo), encontravam-se encerradas ou em processo de insolvência. Estas empresas foram excluídas, ficando a base de dados com 1.510 empresas. No período de recolha de dados, entre novembro de 2011 e fevereiro de 2012, foram recebidos 293 questionários, correspondendo a uma taxa de resposta de 19,40\%, o que se considera bastante satisfatório dado que a taxa de resposta média da gestão de topo se encontra entre $15 \%$ e $20 \%$ (Menon, Bharadwaj, Adidam e Edison, 1999).

Depois de analisar os missing values, a normalidade dos dados e os dados extremos, a amostra ficou representada por 265 observações, correspondendo a uma taxa de resposta de cerca de $18 \%$.

\subsection{ENVIESAMENTO DAS NÃO RESPOSTAS}

Neste estudo a maioria das respostas foram recolhidas após follow-up, realizado por email e telefone. As datas de resposta dos elementos do primeiro grupo situam-se entre 28 de novembro e 14 de dezembro (71 respostas), as respostas do segundo grupo foram recolhidas entre 15 de dezembro a 20 de fevereiro (222 respostas).

Avaliamos o enviesamento das não respostas, através das diferenças entre grupos, comparando as médias das respostas obtidas para os respondentes do primeiro grupo (primeiro quartil) e segundo grupo (quarto quartil) relativamente a todas as variáveis incluídas no modelo. Os resultados mostraram que a maioria das médias das respostas mais tardias são superiores às médias das respostas iniciais, mas as diferenças não são estatisticamente significativas $(p>0.05)$, pelo que o enviesamento das não respostas não é um problema significativo deste estudo (Armstrong e Overton, 1977). 
O tratamento e análise de dados foram realizados com recurso aos programas estatísticos SPSS 19 e LISREL 8.8 .

\section{RESULTADOS}

\subsection{CARACTERIZAÇÃO DA AMOSTRA}

Os resultados desta pesquisa baseiam-se na informação fornecida por 265 empresas exportadoras de várias indústrias da região norte de Portugal. Quanto à dimensão, 207 (78.10\%) das empresas são pequenas e médias empresas (PME), em que 137 (51.70\%) estão representadas por pequenas empresas e $70(26.40 \%)$ por médias empresas. Apenas $23(8.70 \%)$ empresas são de grande dimensão e 35 (13.20\%) são micro empresas. Em relação à experiência na atividade de exportação, verificamos que $49.10 \%$ desenvolvem atividade exportadora há mais de 15 anos e $33.20 \%$ entre 6 a 15 anos, enquanto apenas $4.50 \%$ exportam há menos de 3 anos e $13.20 \%$ entre 3 a 5 anos. No que respeita ao peso das vendas para o mercado externo face às vendas totais, $52,83 \%$ das empresas exportam mais de $40 \%$, enquanto $15.09 \%$ exportam menos de $10 \%, 19.25 \%$ entre $10 \%$ a $24 \%$ e $12.83 \%$ entre $25 \%$ e $39 \%$. As empresas podem expandir a atividade de exportação para diversos mercados e neste estudo verificamos que $39.62 \%$ das empresas vendem para menos de 6 países, $44.15 \%$ entre 6 e 15 países, $5.28 \%$ entre 16 e 25 países e o restante (10.95\%) para mais de 25 países. O principal mercado das empresas respondentes situa-se maioritariamente na União Europeia (72.83\%), seguindo-se países de África cuja língua oficial é o português (16.98\%).

\subsection{RESULTADOS DO MODELO DE EQUAÇÕES ESTRUTURAIS}

Para testar as relações causais entre os diferentes construtos recorremos à análise do modelo de equações estruturais, que constitui a metodologia mais adequada para avaliar relações complexas entre variáveis latentes, medidas por diferentes itens e que se apresentam simultaneamente como variáveis independentes e dependentes. Optamos pela matriz de covariâncias, uma vez que se pretende testar uma estrutura concetual (Hair, Anderson, Tatham e Black, 1998). Bentler, Bagozzi, Cudeck e Iacobucci (2001) referem, por outro lado, que a utilização de uma matriz de correlações pode apresentar testes de qui-quadrado e estimativas de erros-padrão incorretos. A técnica de estimação adotada neste estudo é o método da máxima verosimilhança. Este método é o que se 
Desempenho das Exportações: Influência dos Recursos Intangíveis, Capacidades Dinâmicas e

Orientação Empreendedora

apresenta de utilização mais generalizada (Baumgartner e Homburg, 1996), produzindo estimativas mais fiáveis quando se utiliza matrizes de covariância (Byrne, 1998) e sendo também considerado robusto em relação a violação da normalidade (Diamantopoulos e Siguaw, 2000).

\subsection{AVALIAÇÃO DO MODELO DE MEDIDA}

O modelo de medida foi avaliado em termos de unidimensionalidade, fiabilidade e validade (convergente e discriminante) dos construtos. Os resultados da avaliação do modelo de medida encontram-se na Tabela 1. Nos modelos de primeira ordem, todos os itens reportam de forma significativa ao fator, em termos de loadings e estatísticos, evidenciando desta forma a unidimensionalidade do único fator e todos os loadings das variáveis observadas apresentam valores superiores a 0.70 , demonstrando a existência de validade convergente dos construtos (Garver e Mentzer, 1999). Todas as variáveis latentes apresentam um bom nível de fiabilidade compósita (FC), com valores superiores a 0.60, o que comprova a fiabilidade das escalas (Bagozzi e Yi, 1988). A média da variância extraída (MVE) é superior a 0.50 , fornecendo evidência de validade discriminante dos construtos (Fornell e Larcker, 1981). Nos modelos de segunda ordem (orientação empreendedora), a significância estatística das associações entre os fatores de primeira e segunda ordem é confirmada e os coeficientes excedem o limite mínimo de 0.40, comprovando a validade convergente do construto (Nunnally e Bernstein, 1994) e o quadrado da correlação é menor que a média da variância extraída de cada fator, evidenciado validade discriminante (Fornell e Larcker, 1981).

Tabela 1 - Resultados do modelo de medida

\begin{tabular}{|l|c|}
\hline \multicolumn{1}{|c|}{ CONSTRUTO } & $\begin{array}{c}\text { LOADING } \\
\text { ESTANDARDIZADO }\end{array}$ \\
\hline Relacionamento ( FC=0.90, MVE=0.74) & 0.82 \\
\hline Força do relacionamento existente com clientes. & 0.85 \\
\hline Duração do relacionamento com os nossos distribuidores atuais. & 0.91 \\
\hline Proximidade de relacionamento com os clientes atuais. & 0.91 \\
\hline Conhecimento (FC=0.92, MVE=0.80) & 0.89 \\
\hline Informação sobre o mercado internacional. & 0.87 \\
\hline Conhecimento dos clientes. & 0.87 \\
\hline Conhecimento dos concorrentes. & 0.93 \\
\hline Capacidades dinâmicas (FC=0.95, MVE=0.81) & 0.94 \\
\hline Capacidade de integração de conhecimento externo nas rotinas internas. & 0.86 \\
\hline Capacidade de reconfiguração dos recursos desenvolvidos pela empresa. & \\
\hline Capacidade de integração/combinação dos recursos internos e externos da empresa. & 0.78 \\
\hline Capacidade de resposta ao ambiente em rápida mutação. & 0.82 \\
\hline Orientação Empreendedora & \\
\hline Inovação (FC=0.87, MVE=0.71) & \\
\hline Nos últimos cinco anos, a empresa lançou muitas linhas novas de produtos. & \\
\hline
\end{tabular}




\begin{tabular}{|c|c|}
\hline $\begin{array}{l}\text { Nos últimos cinco anos, as mudanças nas linhas de produto foram em geral muito } \\
\text { significativas. } \\
\text { Proatividade }(\mathbf{F C}=\mathbf{0 . 8 2}, \mathbf{M V E}=\mathbf{0 . 6 4})\end{array}$ & $\begin{array}{l}0.86 \\
0.82\end{array}$ \\
\hline $\begin{array}{l}\text { Relativamente à concorrência, na nossa empresa, normalmente iniciamos ações a que } \\
\text { os concorrentes respondem. } \\
\text { Relativamente à concorrência, na nossa empresa, somos sempre os primeiros a } \\
\text { introduzirem novos produtos/serviços, técnicas de gestão, tecnologias operativas, etc. }\end{array}$ & $\begin{array}{l}0.72 \\
0.80\end{array}$ \\
\hline \multicolumn{2}{|l|}{ Assunção do risco $(\mathrm{FC}=0.87, \mathrm{MVE}=0.71)$} \\
\hline $\begin{array}{l}\text { Em geral, os gestores de topo da nossa empresa, têm uma forte preferência por projetos } \\
\text { de alto risco (com possibilidade de rentabilidades muito altas mas incertas). }\end{array}$ & 0.80 \\
\hline $\begin{array}{l}\text { Em geral, os gestores de topo da nossa empresa, acreditam que, dada a natureza do } \\
\text { mercado, são necessárias ações arrojadas e de grande impacto para alcançar os } \\
\text { objetivos da empresa. }\end{array}$ & 0.93 \\
\hline $\begin{array}{l}\text { Em geral, os gestores de topo da nossa empresa, adotamos uma postura arrojada e } \\
\text { agressiva no sentido de explorar ao máximo a probabilidade de oportunidade } \\
\text { potenciais. }\end{array}$ & 0.78 \\
\hline \multicolumn{2}{|l|}{ Fatores de primeira e segunda ordem } \\
\hline Inovação - Orientação empreendedora & 0.64 \\
\hline Proatividade - Orientação empreendedora & 0.76 \\
\hline Assunção do risco - Orientação empreendedora & 0.57 \\
\hline \multicolumn{2}{|l|}{ Correlaça o entre fatores: } \\
\hline Inovação-Proatividade $\left(\mathrm{R}^{2}=0.41\right)$ & 0.64 \\
\hline Inovação-Assunção do risco $\left(\mathrm{R}^{2}=0.18\right)$ & 0.43 \\
\hline Proatividade-Assunção do risco $\left(\mathrm{R}^{2}=0.20\right)$ & 0.45 \\
\hline \multicolumn{2}{|l|}{ Desempenho das exportações $(\mathrm{FC}=0.92, \mathrm{MVE}=0.79)$} \\
\hline Conseguimos um rápido crescimento nas nossas atividades internacionais. & 0.93 \\
\hline Expandimos as nossas operações nos últimos três anos. & 0.93 \\
\hline Em geral, o desempenho internacional da empresa tem sido muito satisfatório. & 0.81 \\
\hline
\end{tabular}

\subsection{AVALIAÇÃO DO MODELO ESTRUTURAL}

Os resultados mostram um bom ajustamento do modelo $\left(\chi_{(94)}^{2}=154.37\right), p=0.00, \mathrm{CFI}=0.99$, GFI=0.93, NNFI=0.99, RMSEA=0.049). Na tabela 2, apresentam-se os coeficientes estandardizados, valor de $t$ e nível de significância para cada relação postulada no modelo, assim como o coeficiente de determinação para cada construto.

Os resultados mostram que os relacionamentos empresariais influenciam o nível de conhecimento $(\gamma=0.54, t=8.45, p<0.001)$, suportando a $\mathrm{H} 1$. Os relacionamentos e o conhecimento potenciam diretamente e de forma significativa o desenvolvimento de capacidades dinâmicas $(\gamma=0.40, t=6.32, p<0.001 ; \beta=0.40, t=5.92, p<0.001)$, o que permite suportar as H2 e H4. Já o impacto na orientação empreendedora não é significativo pelo que se rejeita a H3 e H5 ( $\gamma=-0.02, t=-$ 0.21 , n.s; $\beta=0.13, t=1,31$, n.s;) As capacidades dinâmicas têm efeito positivo significativo na orientação empreendedora $(\beta=0.36, t=3.43, p<0.001)$, corroborando a H6. Por sua vez, tanto as capacidades dinâmicas como a orientação empreendedora impactam no desempenho das exportações $(\beta=0.21, t=2.42, p<0.001 ; \beta=0.43, t=5.16, p<0.001)$, permitindo suportar as $\mathrm{H} 7$ e $\mathrm{H} 9$. O 
Desempenho das Exportações: Influência dos Recursos Intangíveis, Capacidades Dinâmicas e

Orientação Empreendedora

conhecimento e os relacionamentos não apresentam efeito direto no desempenho das exportações, pelo que rejeitamos as $\mathrm{H} 8$ e $\mathrm{H} 10(\beta=0.12, t=1.56, \mathrm{n} . \mathrm{s} ; \gamma=-0.03, t=-0.40, \mathrm{n} . \mathrm{s} ;)$. Os resultados permitem confirmar seis das dez relações diretas postuladas no modelo proposto.

Tabela 2 - Resultados da avaliação do modelo teórico

\begin{tabular}{|l|c|c|c|}
\hline \multicolumn{1}{|c|}{ PARÂMETROS } & $\begin{array}{c}\text { LOADING } \\
\text { ESTANDARDIZADO }\end{array}$ & $\begin{array}{c}\text { VALOR } \\
\boldsymbol{t}\end{array}$ & $\mathbf{R}^{\mathbf{2}}$ \\
\hline Relacionamentos - Conhecimento & 0.54 & $8.45^{*}$ & 0.29 \\
\hline Relacionamentos - Capacidades Dinâmicas & 0.39 & $5.92^{*}$ & \\
\hline Conhecimento - Capacidades Dinâmicas & 0.40 & $6.32^{*}$ & 0.48 \\
\hline Conhecimento- Orientação Empreendedora & 0.13 & 1.31 & \\
\hline Relacionamentos - Orientação Empreendedora & -0.02 & -0.21 & \\
\hline Capacidades Dinâmicas - Orientação & 0.36 & $3.43^{*}$ & 0.19 \\
\hline Capacidades Dinâmicas - Desempenho das & 0.21 & $2,42^{* *}$ & \\
\hline Conhecimento - Desempenho das Exportações & 0.12 & 1.56 & \\
\hline Orientação Empreendedora - Desempenho das & 0.43 & $5.16^{*}$ & \\
\hline Relacionamentos - Desempenho das Exportações & -0.03 & -0.40 & 0.37 \\
\hline
\end{tabular}

$(*)$ Valor significativo para $p<0.001 ;(* *)$ Valor significativo para $p<0.01$

Os resultados mostram que apesar de o conhecimento e os relacionamentos não apresentarem efeito direto no desempenho das exportações, exercem um efeito indireto através das capacidades dinâmicas. O efeito indireto do conhecimento no desempenho das exportações por intermédio das capacidades dinâmicas é de 0.08 (0.40x0.21). O efeito indireto dos relacionamentos no desempenho das exportações por intermédio das capacidades dinâmicas é de 0.08 (0.39x0.219). Os relacionamentos e conhecimento não apresentarem efeitos diretos e positivos na orientação empreendedora mas indiretos através das capacidades dinâmicas. Por outro lado, os relacionamentos empresariais e conhecimento não influenciam diretamente a orientação empreendedora mas indiretamente através das capacidades dinâmicas, sendo o impacto indireto de 0.14 em ambos os casos $(0.39 \times 0.26 ; 0,40 \times 0.36)$.

\section{DISCUSSÃO E CONCLUSÃO}

A sobrevivência e expansão das empresas e consequente crescimento económico de muitos países estão fortemente dependentes de uma melhor compreensão dos determinantes que influenciam o desempenho internacional (Sousa et al., 2008). O estudo neste campo centra-se sobretudo na forma como os recursos e capacidades permitem às empresas alcançarem vantagem competitiva e desempenho superior em mercados externos. 
Albertina Paula Monteiro, Ana Maria Soares \& Orlando Lima Rua

Com base na RBV e DCV, o presente estudo parte da premissa de que os recursos intangíveis, nomeadamente os relacionamentos empresariais e o conhecimento, influenciam o desenvolvimento de capacidades, tais como capacidades dinâmicas e orientação empreendedora, apresentando um efeito positivo no desempenho das exportações.

Com base na resposta a um questionário online por gestores de topo de 265 empresas exportadoras, verificamos que os relacionamentos empresariais potenciam o desenvolvimento de conhecimento, que estes dois tipos de recursos intangíveis influenciam o desenvolvimento de capacidades dinâmicas não apresentando, no entanto, influência direta na orientação empreendedora. Esta é, no entanto, influenciada de forma indireta por estes recursos através das capacidades dinâmicas. O desempenho das exportações é influenciado positivamente (de forma direta e indireta) pelas capacidades dinâmicas e diretamente pela orientação empreendedora. Estes resultados enfatizam o papel da capacidade dinâmicas e suportam as limitações da teoria RBV para explicar adequadamente o desempenho das organizações (Teece et al., 1997; Eisenhardt e Martin, 2000). Efetivamente, sem a capacidade para integrar, construir e reconfigurar competências internas e externas a fim de responder rapidamente às mudanças do atual ambiente de negócios (Teece, et al., 1997), os recursos intangíveis, não se afiguram como condição suficiente para o desempenho organizacional.

Os resultados deste estudo são consistentes com o estudo de Madsen et al. (2007), com exceção da relação direta entre conhecimento e orientação empreendedora, que não ficou comprovada estatisticamente nesta investigação. Para além de suportar estes relacionamentos num contexto diferente, a presente investigação desenvolve o estudo destes autores, analisando o impacto dos relacionamentos empresariais, do conhecimento e das capacidades no desempenho em contexto internacional, contribuindo assim para o desenvolvimento da literatura e para a prática, revelando-se importante quer para os gestores das empresas exportadoras e para as entidades públicas.

\subsection{LIMITAÇÕES E LINHAS DE INVESTIGAÇÃO FUTURA}

Esta investigação não está isenta de limitações que devem ser consideradas na interpretação e generalização dos resultados. Assinalamos em primeiro lugar limitações relacionadas com o potencial enviesamento provocado pela medida dos dados e pela dimensão da amostra. $\mathrm{Na}$ linha de estudos anteriores, utilizamos escalas de Likert de 1 a 7 pontos para avaliar os construtos, pelo que a maior parte das respostas ao questionário se basearam no julgamento subjetivo dos respondentes. Apesar de se ter identificado em estudos anteriores uma forte utilização de medidas subjetivas para 
Desempenho das Exportações: Influência dos Recursos Intangíveis, Capacidades Dinâmicas e

Orientação Empreendedora

avaliar o desempenho das exportações, é de admitir que algumas respostas não refletem uma situação real do nível dos recursos e capacidades disponíveis para a export venture e do desempenho das exportações. Dado o elevado número de empresas exportadoras optamos por aplicar o questionário às empresas sediadas na região norte de Portugal. Por conveniência, enviamos o questionário apenas às empresas cujo endereço de email nos foi facultado pelo INE (ou recuperado no caso de desatualizado). Embora o email seja hoje uma ferramenta de utilização comum, não podemos generalizar os resultados para a população total.

Entendemos ainda que avaliar as diferentes variáveis deste estudo com base na opinião de um único respondente por empresa pode não refletir exatamente a realidade das empresas, uma vez que as decisões são tomadas por mais de uma pessoa, especialmente nas grandes empresas, que podem ter diferentes opiniões sobre a atividade exportadora (Leonidou e Katsikeas, 1996).

Algumas das limitações apontadas anteriormente poderão constituir linhas futuras de investigação. O estudo ignora os possíveis efeitos mediadores de algumas variáveis (e.g., vantagens competitivas) e efeitos moderadores (e.g., hostilidade do ambiente externo) bem como o efeito de variáveis de controlo, tal como a dimensão da empresa e as características demográficas dos respondentes, o que poderiam possibilitar uma perspetiva adicional para o modelo estudado. Sugerimos ainda analisar as capacidades dinâmicas como um antecedente dos recursos intangíveis, já que neste estudo demonstrou sê-lo em relação à orientação empreendedora. Consideramos ser ainda importante avaliar o modo como os incentivos e apoios governamentais, assim como as parcerias empresariais influenciam diretamente as capacidades e recursos das empresas e indiretamente o desempenho das exportações.

\section{REFERÊNCIAS}

Acedo, F.; Florin, J. (2006) An entrepreneurial cognition perspective on the internationalization of SMEs. Journal of International Entrepreneurship, 4, 49-67.

Avlonitis, G. J.; Salavou, H. E. (2007) Entrepreneurial orientation of SMEs, product innovativeness, and performance. Journal of Business Research, 60, 566-575. 
Amit, R.; Schoemaker, P. J. (1993) Strategic assets and organizational rent. Strategic Management Journal, 14(1), 33-46.

Armstrong, S. J.; Overton, T. S. (1977) Estimating nonresponse bias in mail surveys. Journal of Marketing Research, 16(3), 396-400.

Anand, V.; Glick, W. H.; Manz, C. C. (2002) Thriving on the knowledge of outsiders: Tapping organizational social capital. Academy of Management Executive, 16(1), 87-101.

Arndt, J. (1979) Toward a concept of domesticated markets. Journal of Marketing, 43(4), 69-75.

Bagozzi, R. P.; Yi, Y. (1988) On the evaluation of structural equation models. Journal of the Academy of Marketing Science, 16, 74-94.

Bakar, L. J.; Ahmad, H. (2010) Assessing the relationship between firm resources and product innovation performance: a resource-based view. Business Process Management, 16(3), 420-435.

Barney, J. B. (1991) Firm resources and sustained competitive advantage. Journal of Management, 17(1), 99-120.

Barney, J.; Wright, M.; Ketchen, D. (2001) The resource-based view of the firm: ten years after 1991. Journal of Management, 27(6), 625-641.

Baumgartner, H.; Homburg, C. (1996) Applications of structural equation modeling in marketing and consumer research: a review. International Journal of Research in Marketing, 13, 139-61.

Bentler, P.; Bagozzi, R. P.; Cudeck, R.; Iacobucci, D. (2001) Structural equations modeling - SEM using correlations or covariance matrices. Journal of Consumer Psychology, 10(1/2), 85-87.

Byrne, B. (1998) Structural Equation Modeling with LISREL, PRELIS, and SIMPLIS: basic concepts, applications and programming. Mahwah: Lawrence Erlbaum Associates.

Cavusgil, S. T.; Zou, S. (1994) Marketing strategy-performance relationship: an investigation of the empirical link in export market ventures. Journal of Marketing, 58, 1-21.

Covin, J. G.; Slevin, D. P. (1989) Strategic management of small firms in hostile and benign environments. Strategic Management Journal, 10(1), 75-87.

Davis, D. F.; Mentzer, J. T. (2008) Relational resources in interorganizational exchange: the effects of trade equity and brand equity. Journal of Retailing, 84(4), 435-448.

Dhanaraj, C.; Beamish, P. W. (2003) A resource-based approach to the study of export performance. Journal of Small Business Management, 41(3), 242-261. 
Desempenho das Exportações: Influência dos Recursos Intangíveis, Capacidades Dinâmicas e Orientação Empreendedora

Diamantopoulos, A. ; Siguaw, J. A. (2000), Introducing LISREL. London: Sage Publications.

Eisenhardt, K. M.; Martin, J. A. (2000) Dynamic capabilities: what are they?. Strategy Management Journal, 21(10/11), 1105-1121.

Fornell, C.; Larcker, D. (1981) Evaluating structural equation models with unobserved variables and measurement error. Journal of Marketing Research, 18, 39-50.

Frank, H.; Kessler, A.; Fink, M. (2010) Entrepreneurial orientation and business performance - a replication study. Schmalenbach Business Review, 62, 175-198.

Garver, M. S.; Mentzer J. T. (1999) Logistics research methods: employing structural equation modeling to test for construct validity. Journal of Business Logistics, 20(1), 33-57.

Grant, R. M. (1996) Toward a knowledge-based theory of the Firm. Strategic Management Journal, $17,109-122$.

Gulati, R.; Nohria; N.; Zaheer, A. (2000) Strategic networks. Strategic Management Journal, 21, 203-215.

Hair, J. F.; Anderson, R. E.; Tatham, R. L.; Black, W. C. (1998), Multivariate data analysis. $5^{\text {a }}$ ed. New Jersey: Prentice Hall.

Helfat, C. E.; Peteraf, M. (2003) The dynamic resource-based view: Capability lifecycles. Strategic Management Journal, 24(10), 997-1010.

Henderson R.; Cockburn I. (1994) Measuring competence? Exploring firm effects in pharmaceutical research. Strategic Management Journal, 15, 63-84.

Hitt, M. A.; Hoskisson, R. E.; Kim, H. (1997) International diversification: effects on innovation and firm performance in product-diversified firms. Academy of Management Journal, 40(4), 767-798.

Hung-Hsin, C.; Lee, P.-Y. (2009) The driving drivers of dynamic competitive capabilities: a new perspective on competition. European Business Review, 21(1), 78-91.

Katsikeas, C. S.; Leonidou, C. L.; Morgan, N. A. (2000) Firm-level export performance assessment: review, evaluation, and development. Journal of the Academy of Marketing Science, 28(4), 493511.

Katsikeas, C. S.; Morgan, R. E. (1994) Differences in perceptions of exporting problems based on firm size and export market experience. European Journal of Marketing, 28(5), 17-35. 
Knight, G. A. (1997) Cross-cultural reliability and validity of a scale to measure firm entrepreneurial orientation. Journal of Business Venturing, 12(3), 213-225

Kropp, F.; Lindsay, N. J.; Shoham, A. (2008) Entrepreneurial orientation and international entrepreneurial business venture startup. International Journal Entrepreneurial Behaviour and Research, 14(2), 102-117.

Lages, L. F.; Montgomery, D. B. (2004) Export performance as an antecedent of export commitment and marketing strategy adaptation: evidence from small and medium-sized exporters. European Journal of Marketing, 38(9/19), 1186-1214.

Leonidou, L. C.; Katsikeas, C. S (1996) The export development process: an integrative review of empirical models. Journal of International Business Studies, 27(3), 517-551.

Loane, S.; Bell, J. (2006) Rapid internationalisation among entrepreneurial firms in Australia, Canada, Ireland and New Zealand: An extension to the network approach. International Marketing Review, 23(5), 467-485.

López, S. V. (2005) Competitive advantage and strategy formulation: the key role of dynamic capabilities. Management Decision, 43(5), 661-669.

Lumpkin, G. T.; Dess, G. G. (1996) Clarifying the entrepreneurial orientation construct and linking it to performance. Academic of Management Review, 21(1), 135-172.

Mcdougall, P. P.; Oviatt, B. M. (2000) International entrepreneurship: The intersection of two research paths. Academy of Management Journal, 43(5), 902-908.

Madsen, E. L.; Alsos, G. A.; Borch, O.-J., Ljunggren, E.; Brastad, B. (2007) Developing entrepreneurial orientation: the role of dynamic capabilities and intangible resources. Regional Frontiers of Entrepreneurial Research. Acedido em 15 de novembro 2011, de http://www.swinburne.edu.au/lib/ir/onlineconferences/agse2007/madsen_p94.pdf.

Makadok, R. (2001) Toward a synthesis of the resource-based and dynamic-capability views of rent creation. Strategic Management Journal, 22(5), 387-401.

Menon, A.; Bharadwaj, S. G.; Adidam, P. T.; Edison, S. W. (1999) Antecedents and consequences of marketing strategy making: a model and a test. Journal of Marketing 63(2), 18-40.

Miesenböck, K. J. (1988) Small businesses and exporting: a literature review. International Small Business Journal, 6(2), 42-61.

Miller, D. (1983) The correlates of entrepreneurship in three types of firms. Management Science, 29(7), 770-791. 
Desempenho das Exportações: Influência dos Recursos Intangíveis, Capacidades Dinâmicas e

Orientação Empreendedora

Miller, D.; Shamsie, J. (1996) The Resource-based view of the firm in two environments: the Hollywood film studios from 1936 to 1965 . Academy of Management Journal, 39(3), 519-543.

Morgan, N. A.; Kaleka, A; Katsikeas, C. S. (2004) Antecedents of export venture performance: a theoretical model and empirical assessment. Journal of Marketing, 68, 90-108.

Morgan, N. A.; Vorhies, D. W.; Schlegelmilch, B. B. (2006) Resource-performance relationships in industrial export ventures: The role of resource inimitability and substitutability. Industrial Marketing Management, 35, 621-633.

Nahapiet, J.; Ghoshal, S. (1998) Social capital, intellectual capital, and the organizational advantage. Academy of Management Review, 23(2), 242-266.

Naldi, L.; Nordqvist, M.; Sjöberg, K.; Wiklund, J. (2007) Entrepreneurial orientation risk taking, and performance in family firms. Family Business Review, 20(1), 33-47.

Nunnally, J. e Bernstein, I (1994), Psychometric Theory. $3^{\text {a }}$ ed. New York: McGraw-Hill.

Okpara, J. O. (2009) Entrepreneurial orientation and export performance: evidence from an emergency economy. International Review of Business Research Papers, 5(6), 195-211.

Pearce, J. A., II; Fritz, D. A; Davis, S. (2010). Entrepreneurial orientation and the performance of religious congregations as predicted by rational choice theory. Entrepreneurship Theory and Practice, 34(1), 219-248.

Piercy, N. F.; Kaleka, A.; Katsikeas, C. S. (1998) Sources of competitive advantage in high performing exporting companies. Journal of World Business, 33(4), 278-393.

Sousa, C. M.; Bradley, F. (2006) Cultural distance and psychic distance: two peas in a pod?. Journal of International Marketing, 14(1), 49-70.

Sousa, C. M.; Martínez-López, F. J.; Coelho, F. (2008) The determinants of export performance: a review of the research in the literatures between 1998 and 2005. International Journal of Management Reviews, 10(4), 343-374.

Teece, D. J. (2007) Explicating dynamic capabilities: the nature and microfoundatons of (sustainable) enterprise performance. Strategic Management Journal, 28, 1319-1350.

Teece D. J.; Pisano G.; Amy S. (1997) Dynamic capabilities and strategic management». Strategic Management Journal, 18(7), 509-533.

Tooksoon, P.; Mohamad, O. (2010) Achieving financial export performance: contribution of external resources. International Journal of Marketing Studies, 2(1), 110-116.Tsai, W.; Ghoshal, S. (1998) Social capital and value creation: the role of intrafirm networks. Academy of Management Journal, 41(4), 464-476. 
Vila, N.; Kuster, I. (2007) The importance of innovation in international textile firms. European Journal of Marketing, 41(1/2), 17-36.

Wernerfelt, B. (1984), A resource-based view of the firm. Strategic Management Journal, 5(2), 171-180.

Wiklund, J.; Shepherd, D. (2005) Entrepreneurial orientation and small business performance: a configurational approach. Journal of Business Venturing, 20(1), 71-91.

Wu, L.-Y. (2006) Resources, dynamic capabilities and performance in a dynamic environment: perceptions in Taiwanese IT enterprises. Information e Management, 43, 447-454.

Wu, L.-Y. (2010) Applicability of the resource-based and dynamic-capability views under environment volatility. Journal of Business Research, 63(1), 27-31.

Wu, L.-Y.; Wang, C.-J. (2007), Transforming resources to improve performance of technologybased firms: a Taiwanese empirical study. Journal of Engineering and Technology Management, 24, 251-261.

Zahra, S. A. (2008) Being entrepreneurial and market driven: implications for company performance. Journal of Strategy and Management, 1(2), 125-142.

Zahra, S. A.; Sapienza, H. J.; Davidsson, P. (2006) Entrepreneurship and dynamic capabilities: A review, model and research agenda, Journal of Management Studies, 43(4), 917-955.

Recebido: 12/03/2013

Aprovado: 14/05/2013 